\title{
CASE STUDY OF TEACHER'S ON GOING COGNITION USING VR
}

\author{
Takashi Ikuta $^{1}$, Yasushi Gotoh ${ }^{2}$ and Wataru Uchiyama ${ }^{3}$ \\ ${ }^{1}$ Gifu Women's University \\ 80, Taromaru, Gifu City, Gifu, Japan \\ ${ }^{2}$ Niigata University \\ 8050, Ikarashi 2no-cho, Nishi ward, Niigata City, Niigata, Japan \\ ${ }^{3}$ Niigata Sogo gakuen \\ 494-3, Higashibori-dori, Chuo ward, Niigata City, Niigata, Japan
}

\begin{abstract}
Teacher cognition in classroom lessons is identified by an ongoing method using virtual reality (VR) videos. There are place and time constraints in real time implementation of the ongoing method in synchronous situations. To avoid these, recorded virtual reality videos were used. As a result, the existence of non-corresponding cognition was recognized: teacher educators focused on teaching strategy, while teachers focused on children's individual reactions.
\end{abstract}

\section{KEYWORDS}

$360^{\circ}$ Camera, Teaching Skill, Lesson Cognition, Lesson Research, Ongoing Method

\section{PURPOSE OF RESEARCH}

Dewey (1933) discusses reflection, in a pedagogical context, as the essence of teachers' thinking. Schöne (1983) and Schavelson (1973) also argue from the teacher's classroom teaching context. There are two methods for grasping teachers' reflections :"reflection on action" which considers teachers' reflections after the class and "reflection in action" which considers their reflections in real time during classroom teaching. Since the former does not enable reflection to be grasped during the actual class, real time reflection is especially important and for this the latter i.e. "reflection in action" must be studied.

In specialist field techniques, there are those which can be transmitted using explicit knowledge and those where latent knowledge is involved. In teacher development, reflection in action including latent knowledge is important and an issue in teacher education. In teacher education although there is research (Walshe and Driver, 2019) into using video recordings for teacher training, there are few practical studies focusing on this issue as it concerns teachers' cognition. In this study the authors propose the ongoing method (OGM) with VR recorded video as a tool for understanding teachers" "reflection in action."

\section{METHOD}

\subsection{OGM}

OGM is a method that enables us to understand lesson cognition in the classroom in real time (Ikuta, 1999). In this study the authors define lesson cognition as the cognition of the decision-making process. Teachers regard a teaching event as consisting of cognition, judgment and action. The OGM method involves a process in which

1. Observers express vocally and record their cognition of the classroom lesson process in real time. 
2. After the lesson, the utterances of the observers are recorded synchronously with the classroom events and their personal interpretation is described.

3. The cognition of the observers is compared and analyzed and the teachers's cognition and reflection are described.

OGM makes it possible to compare and discuss for the same lesson the real time lesson cognitions of multiple observers.

In OGM, teaching events in which multiple observers participate are divided into "cognition of different teaching events' and cognition of same teaching event." Since observers' cognitions may differ even if they are watching the same event, the features of the cognition can be clarified by dividing cognition of the same teaching event into corresponding cognition where the object and contents of the cognition are the same, and non-corresponding cognition where the contents are different. In cognition of different teaching events, the object of the observers' cognition is itself different.

OGM using video recordings means that observers can freely select place and time, but their main advantage lies in the selectivity of the field of view. In this case, a VR video was watched and utterances made according to an ongoing protocol with the objective of synchronous understanding and comparing of VR screens. This is a new method of classroom observation in which multiple observers freely record, analyze and compare their own cognition with that of other people under asynchronous conditions,

On the other hand, although courses from scenarios do exist for teacher training using VR, virtually no research uses the present approach.

\subsection{Classroom Lesson VR and Participating Observers}

The VR lesson scenario used was arithmetic (round numbers) 54 minute lesson at $\mathrm{J}$ elementary school Year 4. Two persons, a teacher educator and a teacher were present. The observer put on a VR head set, observed the lesson and recorded utterances by ongoing process (OGP). The date was November 2017. The field of view in the VR and the OGP were synchronously recorded and played back and the lesson protocol and utterances synthesized into a new scenario to be used as basic data.

\section{RESULTS}

\subsection{Corresponding Cognition and Non-Corresponding Cognitions (OGP in Classroom Process)}

The teacher educator made 51 utterances and the teacher 135. The classroom lesson was divided into introduction, main content and summary. Teaching-learning events were classed from A to M. Viewers cognitions in relation to each event were sorted into corresponding cognition and non-corresponding cognition (Table 1).

As shown in Table 1, introduction events A and B were non-corresponding cognition, and while non-corresponding cognition was intermingled in the main content stage, mid-level main content events $\mathrm{G}, \mathrm{H}$ and I were continuously non-corresponding cognition. Hence there were differences in observers' cognition in relation to the main content which is the intermediate heart of the lesson. 
Table 1. Viewers cognitions in relation to each event

\begin{tabular}{|c|c|c|c|}
\hline \multirow{2}{*}{ Lesson Process } & $\begin{array}{c}\text { Teaching- } \\
\text { learning event }\end{array}$ & $\begin{array}{c}\text { Corresponding } \\
\text { cognition }\end{array}$ & $\begin{array}{c}\text { Non-corresponding } \\
\text { cognition }\end{array}$ \\
\cline { 3 - 4 } & Introduction & & \\
& A & & \\
\hline \multirow{5}{*}{ Main content } & C & & \\
& D & & \\
& E & & \\
& F & & \\
& G & & \\
& H & & \\
& I & & \\
& J & & \\
\hline Summary & K & & \\
\hline
\end{tabular}

\subsection{Changes in Number of Utterances Depending on Teaching-Learning Event}

The number of utterances per teaching-learning event is shown in figure 1 . There were no radical differences in overall trend between the observers. As shown in the ratio for each teaching-event in relation to total number of individual utterances, the difference in frequency ratio between the two observers is large in introduction event A, main content events F, G and J and summary event $\mathrm{M}$. There was non-corresponding cognition for $\mathrm{G}$ and $\mathrm{J}$ in events where the difference was large.

Looking at non-corresponding cognition in event $\mathrm{G}$, the teacher educator predicted from an early stage that since the first step of dealing with round numbers was insufficiently understood, this lack of understanding would affect the second step of estimation and pointed this out as a structural guidance issue. The teacher educator consistently saw the basic guidance framework as a problem.

Teacher educator's OGPs are as follows.

- "It looks as though the children add up the whole 3000 yen to see whether they have enough money or not. This means that the first step, which should be to understand round numbers, can't be used to learn about approximate estimations."

- "The fact that for the first step we have not consolidated former learning task has an impact."

- "Here we see that most children add up all the numbers. Clearly, they are not making an estimation."

On the other hand, the teacher was interested in what the children were thinking at various times, pointing out the link between ambiguity in the intention of the teacher's questions and children's understanding. The teacher focused on each child's individual understanding.

Teacher's OGPs are as follows.

- "In the question, "Will it all be enough?" what are the children thinking?"

- "Are children able to understand words and formulae?"

- "The child ends up not knowing what to do."

In the summary stage, although the number of statements of both participants varied greatly, both pointed out the fact that the notion of approximate estimation was not well understood by the children (corresponding cognition). 


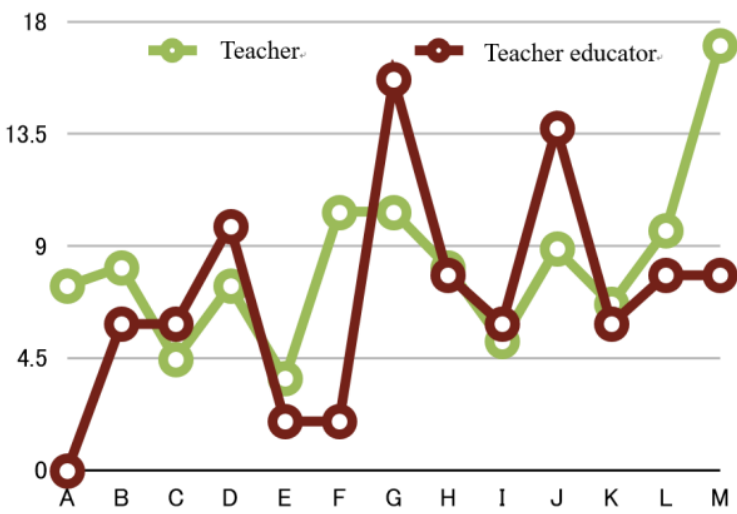

Figure 1. The number of utterances per teaching-learning event

\section{DISCUSSION AND SUGGESTIONS}

In these above case studies,

1) OGM with VR recorded video enables us to grasp observers' reflections in action and show that real time cognition and reflection are different.

2) These findings can provide clues about future teacher training methods because in classroom study, what participants see, their judgments and reflections, are important elements.

Looking at students and novice teachers, it becomes possible to create a method which studies classroom lesson techniques with VR images where young people compare their cognition with the cognition of experienced teachers and use differences as a clue.

3) Asynchronous teacher training with VR recorded video can be carried out in a form that is very close to real time classroom participation (Ikuta, et al., 2018) so that the immersion experience can be re-experienced thanks to VR and contribute to teacher development by its intermediary function in relation to the classroom.

\section{ACKNOWLEDGEMENT}

This work was supported by JSPS KAKENHI Grant Number 18H01061 and 19H01712.

\section{REFERENCES}

Dewy, J. 1933. How we think: A restatement of the relation of reflective thinking to the educative process. Heath and Company, New York, USA.

Ikuta, T. 1999. “Jugyo wo Tennkaisuru Chikara”, in Asada. et (ed.) Seicho suru Kyoshi, Kaneko Shobou, Tokyo, Japan, pp. 42-54. (In Japanese)

Ikuta, T. \& Uchiyama, Y. 2018. $360^{\circ}$ camera niyoru Jugyokiroku no katuyou to igi. Technical report of Gifi women's University Digital archive institute. Vol. 3, No. 2, pp. 29-34. (In Japanese)

Shavelson, R.J. 1973. The basic teaching skill: Decision making Research and Memorandum.104. Stanford Center for Research and Development in teaching.

Schöne, D. 1983. The Reflective Practitioner: How Professionals Think in Action. Basic Books, New York, USA

Theelen, H., Beemt, A. \& Brok, P. 2019. Using 360-degree videos in teacher education to improve preservice teacher's professional interpersonal vision. Journal of Computer Assisted Learning. 2019, pp. 1-9.

Reyna J. 2018. The Potential of 360-Degree Videos for Teaching, Learning and Research, Proceedings of INTED2018 Conference 5th-7th March 2018. Valencia, Spain, pp. 1448-1454.

Walshe, N. \& Driver, P. 2019. Developing reflective trainee teacher practice with 360-degree video. Teaching and Teacher Education: An International Journal of Research and Studies, Vol. 78, No. 1, pp 97-105. 